(C) [2010] IEEE. Reprinted, with permission, from [Wenjing Jia, Xiangjian He and Qiang Wu, ECCH: A novel color coocurrence histogram, EAcoustics Speech and Signal Processing (ICASSP), 2010 IEEE International Conference on, 14-19 March 2010]. This material is posted here with permission of the IEEE. Such permission of the IEEE does not in any way imply IEEE endorsement of any of the University of Technology, Sydney's products or services. Internal or personal use of this material is permitted. However, permission to reprint/republish this material for advertising or promotional purposes or for creating new collective works for resale or redistribution must be obtained from the IEEE by writing to pubs-permissions@ieee.org. By choosing to view this document, you agree to all provisions of the copyright laws protecting it 


\title{
ECCH: A NOVEL COLOR COOCURRENCE HISTOGRAM
}

\author{
Wenjing Jia, ${ }^{1}$ Xiangjian He, ${ }^{1,2}$ and Qiang $\mathrm{Wu}^{1}$ \\ ${ }^{1}$ Centre for Innovation of IT Services and Applications (iNEXT) \\ University of Technology, Sydney, Australia \\ ${ }^{2}$ Department of Electronic and Information Engineering \\ Hong Kong Polytechnic University, Hong Kong
}

\begin{abstract}
In this paper, a novel color cooccurrence histogram method, named $e C C H$ which stands for color cooccurrence histogram at edge points, is proposed to describe the spatial-color joint distribution of images. Unlike all existing ideas, we only investigate the color distribution of pixels located at the two sides of edge points on gradient direction lines. When measuring the similarity of two eCCHs, the Gaussian weighted histogram intersection method is adopted, where both identical and similar color pairs are considered to compensate color variations. Comparative experimental results demonstrate the performance of the proposed eCCH in terms of robustness to color variance and small computational complexity.
\end{abstract}

Index Terms - spatial-color joint distribution, image similarity, license plate

\section{INTRODUCTION}

Color histograms have been widely used as a stable representation over changes in view for image retrieval and object detection. Traditional histogram-based approaches, such as the one proposed by Swain and Ballard in [1], did not take into account the geometric relationships between pixels, and therefore they are likely to produce false positives because two apparently different images may have same color histograms. Funt and Finlayson [2] used histograms of the color ratios of neighboring pixels, and hence introduced a slight amount of spatial information in their histogram representation. Gevers [3] further developed the color ratio gradient (CRG) to make it insensitive to geometry and position of objects, shadow, illumination, and other imaging conditions.

Recently, more efforts have been made to incorporate spatial information into color distribution. Pass and Zabih [4] defined the color coherence vectors to compare the number of coherent pixels and the number of non-coherent pixels of same colors in a model image and a target image. A more attractive approach is to use the color cooccurrence histograms (CCHs), also called the color correlograms in [5]. Chang

This work was supported by the UTS CPRF program. and Krumm [6] originated an object detection algorithm using the $\mathrm{CCHs}$. The $\mathrm{CCH}$ records the number of pairs of colored pixels that are separated at certain distances in an image space. In [7], Crandall and Luo proposed a more flexible spatial-color joint probability function, called the color edge cooccurrence histogram (CECH). The $\mathrm{eCCH}$ is computed for points on the boundaries of two neighboring regions.

Above histograms constrain the spatial relationships of the pairs of colored pixels in an object, but allow for some degrees of distortion. When applied for image retrieval [5], partially occluded objects recognition [6] and non-rigid color object detection [7], desirable experimental results are obtained.

In this paper, a novel spatial-color joint distribution, named color cooccurrence histogram at edge points (abbreviated as $e C C H$ ), is proposed for measuring image similarities. The innovations and benefits of our idea are mainly in the following three aspects. (1) Unlike the $\mathrm{CECH}$ algorithm proposed in [7], our eCCH only cares about the color distribution near edge points. This not only improves the computation efficiency but also prevents solid color regions from dominating the histogram [7]. (2) Our eCCH is only computed in regard to the pairs of pixels which are located on two sides of edge points along gradient direction lines. This makes the resultant $\mathrm{eCCH}$ invariant to rotation and deformation of objects in images. (3) Instead of using the conventional methods to measure the similarities between two eCCHs, we extend and adopt the Gaussian weighted histogram intersection (GWHI) method [8] to take into account the matchings between both identical color pairs and similar color pairs.

Our goal is to develop a general algorithm for matching various compound color object images, such as car license plates, road signs, logos, etc., which have a limited set of multiple colors arranged in specific spatial layouts. The appearance of such color object images may vary dramatically from object to object and from scene to scene.

The remaining parts of the paper are organized as follows. Section 2 gives the details about computing eCCH and the distance between two eCCHs. In Section 3, the proposed algorithm is tested and comparative experimental results are given. The paper concludes in Section 4. 


\section{COLOR COOCCURRENCE HISTOGRAM AT EDGE POINTS (ECCH)}

\subsection{Computing eCCH}

Step 1: Detecting Edges. The first step is to obtain the edge map $E$ of input image $F=f(x, y)$. Color edge detection has been a classical topic in the area of color image processing for many years. In the original CECH method [7], this is done using the simple color pixel difference. In [9], a more accurate representation of edges is obtained using vector-order statistics method. Since it is still hard to obtain a fine single-pixelwidth edge map, instead we use the pseudo-edge points which have relatively strong gradient intensities. In other words, pixels that have a gradient intensity stronger than a predefined threshold, denoted by $T H_{G}$, are viewed as "edge" pixels and the color distribution nearby will be investigated. In order to do so, a grey-level copy of the input color image is obtained first. The classic Sobel operator [10] is then used to obtain the first-order partial derivative $G_{x}$ and $G_{y}$ of the grey-level intensity for its simpleness.

Step 2: Locating Pairs of Pixels. For each edge point $e(x, y)$ (abbreviated as $e$ for simplification), two points, denoted by $p_{1}\left(x_{1}, y_{1}\right)$ and $p_{2}\left(x_{2}, y_{2}\right)$ respectively, are taken. The two points are located on the two sides of the edge point $e$ on its gradient direction line with a fixed distance, denoted by $d$, away from the reference edge pixel. Denote the gradient angle at the edge point $e(x, y)$ as $\theta(x, y)$ (simplified as $\theta$ ), the coordinates of the pixel pair $p_{1}$ and $p_{2}$, can be computed as

$$
\left\{\begin{array}{l}
x_{1}=x-d \times \cos \theta \\
y_{1}=y+d \times \sin \theta
\end{array}\right.
$$

and

$$
\left\{\begin{array}{l}
x_{2}=x+d \times \cos \theta \\
y_{2}=y-d \times \sin \theta
\end{array},\right.
$$

where the gradient angle $\theta$ can be computed as

$$
\theta(x, y)=\tan ^{-1}\left(\frac{G_{y}}{G_{x}}\right) .
$$

Step 3: Collecting eCCH and Normalizing. Denote the color values at points $p_{1}$ and $p_{2}$ as $\vec{c}_{1}$ and $\vec{c}_{2}$ respectively, the corresponding bin $h\left(\vec{c}_{1}, \vec{c}_{2}, d\right)$ of eCCH is counted as

$$
\begin{aligned}
& h\left(\vec{c}_{1}, \vec{c}_{2}, d\right)= \\
& \operatorname{size}\left(\left\{\left(p_{1}, p_{2}\right) \mid \begin{array}{c}
p_{1}, p_{2} \in F, \text { and } \\
\left\|p_{1}-p\right\|=\left\|p_{2}-p\right\|=d
\end{array}\right\}\right), \vec{c}_{1}, \vec{c}_{2} \in \mathbf{C}
\end{aligned}
$$

where $\mathbf{C}$ is the color set of the image, and the function size returns the total number of elements in a set.

All edge points in the whole image plane are scanned. The frequency of occurrences of the same color pairs in the image with above spatial constraint is recorded and summed in the corresponding bin of the eCCH. Finally, the eCCH is normalized to the range of $[0,1]$ using the total number of edge points in the image.

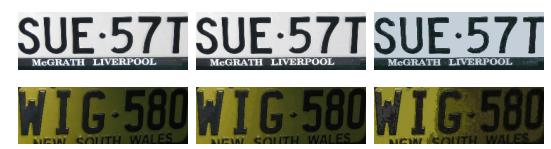

Fig. 1. Original license plate images (left) and their quantized counterparts using the variance minimization algorithm (middle) and the color merging method (right).

\subsection{Color Quantization}

It is known that the total number of colors for a 24-bit truecolor image is $2^{24}$. Without reducing the number of colors, it is unlikely that the histogram comparison operation could be executed in real-time with such a tremendous dimension.

The operation for reducing color number is also called color quantization in references. In [6], color quantization was simply performed in RGB color space using a $k$-means nearest neighbor algorithm. In [7], a perceptual color naming method was employed in order to handle color variation. The standard ISCC-NBS Color Names Dictionary [11] was employed to quantize and name the color space. Since the colors of compound color objects, such as vehicle license plates, only take a very small part of the standard color set, such quantizing methods usually cause large perceptible difference in quantized images and even introduce fake edges (see the right column of Figure 1).

In our method, Wu's [12] optimal color quantization algorithm, which is based on variance minimization, is employed to reduce the number of colors by introducing only slight color distortion into the quantized images. Figure 1 gives some examples of color quantization results obtained using two methods.

Eight and 16 colors have been tested to quantize number plate images. Since using 16 colors did not make much improvement for image matching results, each image is quantized into eight colors using Wu's variance minimization algorithm. Note that exactly which colors are referred in each quantized image depend on its original color distribution.

\subsection{Similarity Measure}

After the eCCH is obtained, the next problem is how to compare two histograms.

Normalised $L_{1}$-norm distance and the histogram intersection (HI) [1] have been proposed in the past for comparing histograms. However, both of the metrics demand an exact bin-to-bin identical color matching between histograms and thus are very sensitive to the color distortion caused by various illumination conditions under which the images were captured. In practice, the colors of real world images can be distorted both in the scene itself and in the image capturing process. Hence, images with same visual information but with different color intensities may degrade the similarity level significantly when the conventional $\mathrm{HI}$ is used. 
In order to overcome this problem, Wong et al. [13] proposed a merged-palette histogram matching (MPHM) method. The essence of the method is to extend the intersection from bins of identical colors to bins of similar colors. In their algorithm, as long as the distance between two colors is less than a fixed threshold, say $T h$, the intersection between the bins of these two colors will be calculated. This algorithm has produced more robust image retrieval results for images captured under various illumination conditions. However, it assumes an identical weight of the contribution between colors which have different similarities with the given color. When applied to license plate image matching, the algorithm exhibited to be very sensitive to color variations.

In [8], a Gaussian weighted histogram intersection (GWHI) method has been proposed which overcomes the above problems and has demonstrated to be much less sensitive to color variations compared with the conventional HI method and the MPHM method [8]. In this method, instead of applying identical weights to different colors, a Gaussian weight function of color distance has been employed to differentiate the matching between colors which have different distances to the given color.

For the purpose of comparing two eCCHs, where there are two pairs of colors involved in the intersection, the GWHI method is further extended to facilitate intersection of two pairs of colors. The larger distance of two color pairs is taken to decide the Gaussian weight function. The reason for this is that for any two pairs of colors in two images respectively, as long as there is one pair of colors being viewed as "dissimilar", the eCCH between these two pairs of colors should not be matched. This design further guarantees that the intersection between eCCH bins only happens on "similar" color pairs with the condition that both color pairs must be viewed as "similar" before an intersection is computed.

\section{EXPERIMENTS}

To demonstrate the advantages of eCCH and suggest potential other applications, experiments are designed where the eCCH is used for classifying vehicle license plate images based on their major color schemes.

Besides character information, the color information in license plates also plays an important role in identifying them as unique ones. License plates in some countries use colors to show the types of vehicles. The colors used in license plates tend to bear important information which should be used with characters in license plates for a unique identification.

In this experiment, the proposed $\mathrm{eCCH}$ algorithm is tested on vehicle license plate images for color-based license plate classification. The aim is to find a robust classification between different classes of license plate images. Two license plate images are viewed to belong to the same class when they have similar foreground and background colors, but they may have quite different contents (characters), sizes, orienta- tions and viewing conditions. Images of two classes of vehicle license plates are tested. They are plates with yellow background and plates with white background, as shown in Figure 2. All number plate images in experiments are with dimension of $120 \times 40$. A yellow license plate image is selected as the model image for yellow class, which is shown in the first row of Figure 2. The selection criterion is that the intensity of the model image should not be too strong or too weak compared with other images.

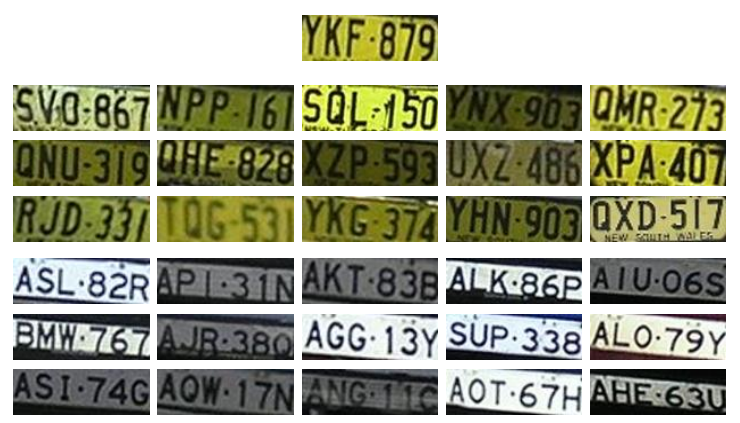

Fig. 2. The yellow model image (the first row) and some examples of yellow (the second to sixth rows) and white target images (lower three rows) used in our experiments.

To show the superiority of the proposed method over three other methods, i.e., the conventional HI method, Wong's MPHM method, and Gevers' CRG method, classification results on 87 yellow plates and 43 white plates using four different methods are plotted in Figure 3. The average time used for computing matching using these four methods are given in Table 1. All experiments are performed on a PC with Intel Core 2 2.4GHz CPU and 3.0GB RAM.

Table 1. Average time used for computing image matching between two license plate images using the four methods.

\begin{tabular}{|c|c|}
\hline Methods & Time (in milliseconds) \\
\hline Conventional HI [1] & 271 \\
\hline Wong's MPHM [13] & 294 \\
\hline Gevers' CRG [3] & 1 \\
\hline Our proposed eCCH & 6 \\
\hline
\end{tabular}

As seen in Figure 3(a) that, using the conventional HI method, it is almost impossible to separate two classes of number plate images via their color histograms. Using the MPHM method, this has been improved a lot. As shown in Figure 3(b), the matching rates between same class of number plates (inter-class) have all been increased, while those between different classes of number plates (inter-class) are still very low. However, as seen from the figure, the matching rates of same class of plates (intra-class) still spread in a wide range. Using Gevers' CRG method, a more stable 


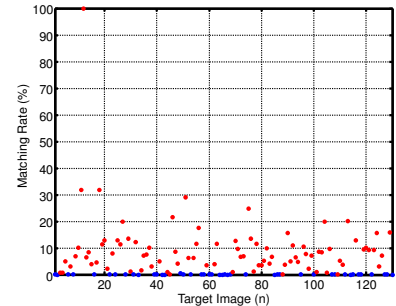

(a) using the conventional HI method

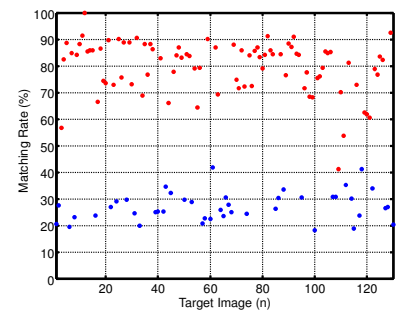

(c) using the CRG method

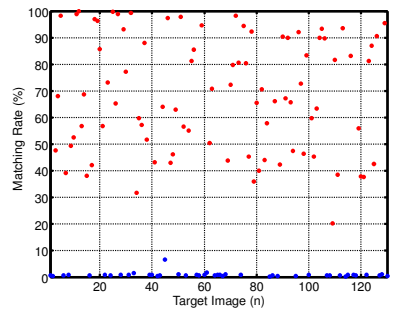

(b) using the MPHM method

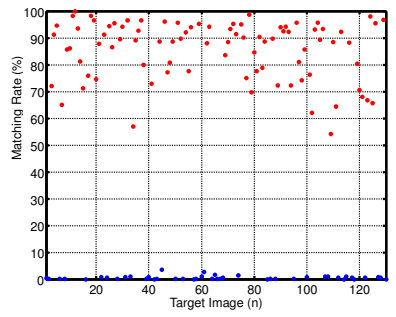

(d) using the proposed eCCH method
Fig. 3. Histogram matching results obtained using four different methods. Matching data of yellow license plate images are marked as red dots, and matching data of white license plate images are marked as blue dots (darker if viewed in black-white picture).

matching results are shown in Figure 3(c) for both same class of plates and different classes of plates. Using the proposed eCCH method, a much better classification performance can be obtained which is shown in Figure 3(d). Also note that, computing matching using the conventional $\mathrm{HI}$ method and MPHM method needs around 0.3 seconds in average, while using eCCH it takes only six milliseconds on average.

\section{CONCLUSIONS}

In this paper, a novel spatial-color joint distribution, $\mathrm{eCCH}$, is proposed for measuring the similarities between images. The advantages of the eCCH in terms of robustness to color variations and computation efficiency is shown in experiments where the eCCH is used for color-based license plate classification. Comparative experimental results demonstrate the superiority of the proposed method over other three methods.

\section{REFERENCES}

[1] Michael J. Swain and Dana H. Ballard, "Color indexing," International Journal of Computer Vision, vol. 7, no. 1, pp. 11-32, 1991.
[2] B.V. Funt and G.D. Finlayson, "Color constant color indexing," IEEE Transactions on Pattern Analysis and Machine Intelligence, vol. 17, no. 5, pp. 522-529, 1995.

[3] T. Gevers and W.M. Smeulders, "Color constant ratio gradients for image segmentation and similarity of texture objects," Proceedings of the 2001 IEEE CVPR, vol. 1, pp. 18-25, 2001.

[4] Greg Pass and Ramin Zabih, "Histogram refinement for content-based image retrieval," Proceedings of the 3rd IEEE WACV, pp. 96-102, 1996.

[5] Jing Huang, S.R. Kumar, M. Mitra, Wei-Jing Zhu, and R. Zabih, "Image indexing using color correlograms," Proceedings of the 1997 IEEE CVPR, pp. 762-768, 1997.

[6] Peng Chang and J. Krumm, "Object recognition with color cooccurrence histograms," Proceedings of the 1999 IEEE CVPR, vol. 2, pp. 498-504, 1999.

[7] D. Crandall and Jiebo Luo, "Robust color object detection using spatial-color joint probability functions," Proceedings of the 2004 IEEE CVPR, vol. 1, pp. 379385, 2004.

[8] W. Jia, H. Zhang, X. He, and Q. Wu, "Gaussian weighted histogram intersection for license plate classification," Proceedings of the 18th ICPR, pp. 574-577, 2006.

[9] Raymond Phan, John Chia, and Dimitrios Andtroutsos, "Unconstrained logo and trademark retrieval in general color image databases using color edge gradient co-occurrence histograms," Proceedings of the 2008 ICASSP, pp. 1221-1224, 2008.

[10] R. C. Gonzalez and R. E. Woods, Digital image processing, Prentice Hall, New Jersey, 2002.

[11] Kenneth L. Kelly and Deane B. Judd, "Color universal language and dictionary of names," National Bureau of Standards special publication 440. Washington, DC: U.S. Department of Commerce, National Bureau of Standards, 1976.

[12] Xiaolin $\mathrm{Wu}$, "Efficient statistical computations for optimal color quantization," Graphics Gems, vol. 2, pp. 126-133, 1991.

[13] Ka-Man Wong, Chun-Ho Cheung, and Lai-Man Po, "Merged-color histogram for color image retrieval," Proceedings of the 2002 ICIP, vol. 3, pp. 949-952, 2002. 